\title{
An autologus lower limb prosthesis
}

\author{
P R Nanayakkara ${ }^{1}$, K G Karunarathna ${ }^{1}$, T Gobyshanger ${ }^{1}$ and M V Perera ${ }^{1}$
}

(Index words: femur, tibia, osteosarcoma, amputation, limb rotationplasty)

\section{Introduction}

Van Nes rotationplasty is a reconstructive and salvage procedure of the lower limb. The technique replaces the amputated thigh and knee with an autologus biological 'prosthesis', the patient's own ipsilateral ankle, which is rotated 180 degrees and fixed at the level of the contralateral knee. This procedure was first adapted by Borggreve of Germany in 1930 to treat a knee joint destroyed by tuberculosis and it was modified by Van Nes in 1950 to treat patients with congenital limb deficiencies [1]. The Van Nes procedure converts a potential transfemoral amputee to a functional transtibial amputee.

\section{Case report}

A 48-year old man presented with a painful swelling in the left thigh of two months duration. A plain radiograph and CT scan of the femur showed a lytic lesion in the midshaft (Figure 1). Considering his age, site of the lesion and the short duration of symptoms, a probable diagnosis of a bony metastasis was made. As the investigation to identify a primary site failed, and there was an impending pathological fracture, it was decided to obtain a tissue diagnosis and stabilise the fracture. At open biopsy, the tumour was found to be extending into the surrounding soft tissues. Excision of the tumour and internal fixation was done with a plate and screws, after reinforcing with bone cement (Figure 2). The histology of the excised tumour showed a telangiectatic osteosarcoma.

Following a course of chemotherapy, a curative surgical procedure was planned as there were no distant metastases. After discussing the advantages and disadvantages of the two available surgical options, the Van Nes procedure was preferred over hip disarticulation by the patient.
After preoperative planning, surgery was carried out under general anaesthesia. A fish mouthed incision over, and a circumferential incision in the proximal leg constituted the respective skin resection margins. After dissecting the femoral vascular bundle and the sciatic nerve along its length (Figure 3 ), the femur was divided $7 \mathrm{~cm}$ distal to the lesser trochanter and tunnelled into the tibial medullary cavity after the latter bone was divided at the level of the tibial tuberosity. Osteosynthesis was achieved with an intramedullary nail.

The postoperative period was uneventful and physiotherapy of the 'new knee' was started. Two months after surgery the patient is being followed up by the oncologist for a second course of chemotherapy. He has no clinical or radiological evidence of residual local or distant malignant disease. He walks on a temporary prosthesis until a custom made prosthesis is available (Figure 4).

\section{Discussion}

Rotationplasty is a reconstructive option that provides a highly functional limb and is a better option than an above-knee amputation or a hip disarticulation because patients have better control of their prosthetic knee [2, 3]. The indications for this procedure are osteosarcoma of the femur and proximal tibia, congenital absence of the femur and destruction of the knee joint as in trauma or severe burns $[1,3,4]$.

The Van Nes procedure is preferably performed under general anaesthesia. The surgical procedure involves identification and preservation of the femoral vascular bundle and the sciatic nerve. The quadriceps tendon is sectioned, together with the vastus medialis and lateralis. The femur and the proximal tibia are then divided as planned and the intervening resected tibia is removed and

${ }^{1}$ Department of Orthopaedics, National Hospital of Sri Lanka, Colombo.

Correspondence: PRN, e-mail <pubudu_randima@hotmail.com>. Received 28 October 2008 and revised version accepted 20 April 2009. Competing interests: none declared. 
submitted for pathological examination [3]. The distal segment that includes most of the leg and the ankle is rotated through 180 degrees and osteosynthesis is carried out. The nerve and vessels are coiled in between the groups of muscles to avoid acute kinks. The thigh muscles are attached to those of the leg, the biceps femoris and semimembranosus to the tibialis anterior tendon, and the quadriceps tendon is attached to the gastrocnemius and the soleus muscles. The ankle is placed in such a way that it comes to lie at the same level as the contralateral knee.

The quality of life of patients that have undergone Van Nes rotationplasty is comparable to those who have an endoprosthesis and it is better than those who have an above-the-knee amputation. Rotationplasty is a favourable alternative to amputation or endoprosthetic replacement, either as a primary or as a salvage procedure [3].

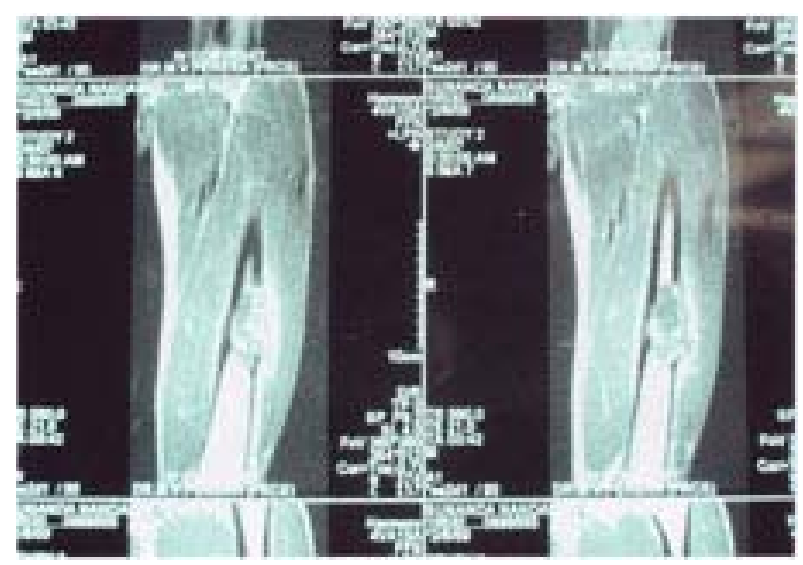

Figure 1. CT scan of the femur showing a lytic lesion in its mid shaft.

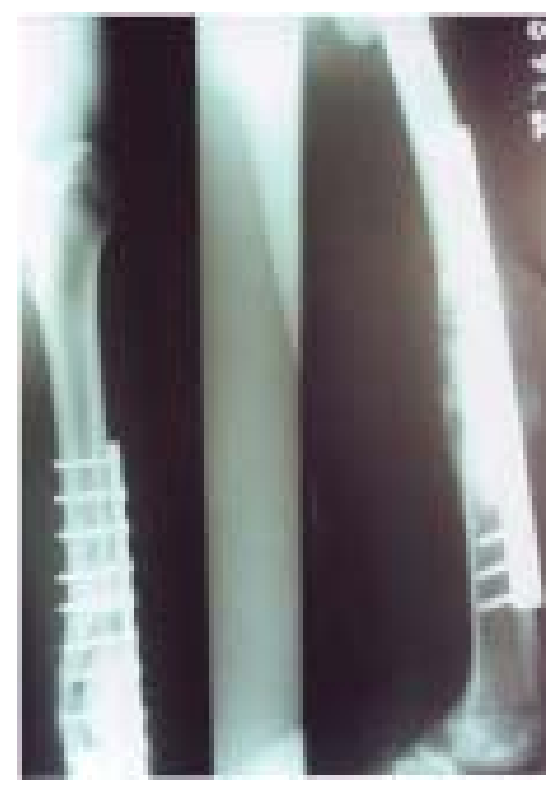

Figure 2. Left femur internally fixed with a plate and screws.

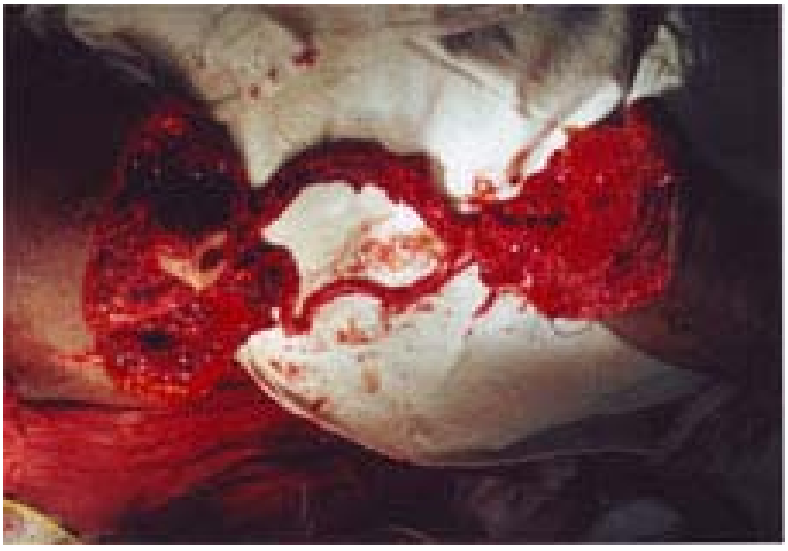

Figure 3. Preservation of the femoral vascular bundle and the sciatic nerve.

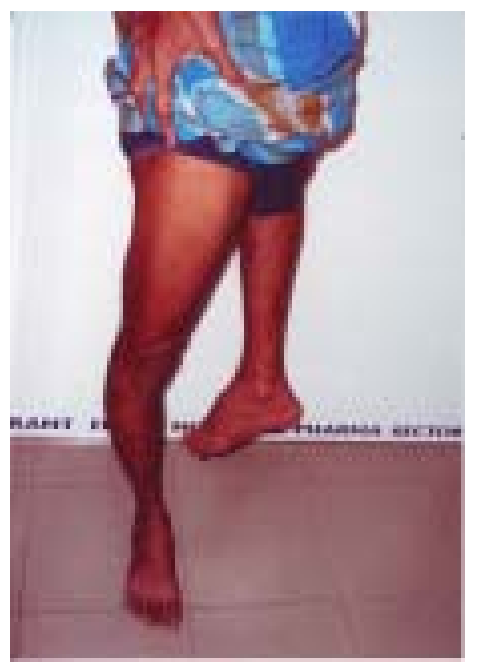

Figure 4. After Van Nes procedure, the ankle comes to lie at the same level as the contralateral knee.

\section{References}

1 DeBari A, Krajbich JI, Langer F, et al. Modified Van Nes rotationplasty for osteosarcoma of the proximal tibia in children. Journal of Bone and Joint Surgery [Br] 1990; 72: 1065-9.

2 Krajbich I, Bochmann JD. Van Nes rotationplasty in tumour surgery. In: Bowker HK, Michael JW eds. Atlas of Limb Prosthetics: Surgical, Prosthetic, and Rehabilitation Principles. Rosemont IL: American Academy of Orthopaedic Surgeons, 1992: Chapter 36 [http://www.oandplibrary.org/ alp/chap36-02.asp].

3 Cammisa FP, Glasser DB, Otis JC, et al. The Van Nes tibial rotationplasty. A functionally viable reconstructive procedure in children who have a tumor of the distal end of the femur. Journal of Bone and Joint Surgery 1990; 72: 1541-7.

4 Khatri B, Richard B. Use of Van Nes rotationplasty to manage a burnt knee. Burns 2000; 26: 88-91. 